\title{
Review on Tendon and Ligament Repair Techniques
}

\author{
Gebremedhin Yohannes ${ }^{1 *}$ and Guesh Negash ${ }^{2}$ \\ ${ }^{1}$ College of Veterinary Medicine, Hawassa University, Ethiopia \\ ${ }^{2}$ College of Veterinary Medicine, Mekelle University, Ethiopia
}

Received: June 18, 2018; Published: June 26, 2018

*Corresponding author: Gebremedhin Yohannes, College of Veterinary Medicine, Hawassa University, P0 Box 5, Hawassa, Ethiopia

\begin{abstract}
Tendons and ligaments are similar dense fibrous connective tissues that connect skeletal muscle to the skeletal elements (bone) and bone to bone, respectively. The main function of ligaments and tendons is to transmit tensile loads. The most commonly damaged tendons and ligaments are those that run down the back of the limbs from the stifle/elbow to the hoof (the superficial digital flexor tendon, the deep digital flexor tendon, the accessory ligaments and the suspensor ligament). The objective of this seminar paper is to review tendon and ligament repair techniques. Most tendon strains are associated with excessive loading and overstretching of the tendon due to excessive physical stress. The end result of tendon and ligament injury is inflammation which manifested in pain, lameness, heat and swelling (typical 'bowed' appearance). Ultrasound is an excellent diagnostic tool that allows veterinarian to view the fiber pattern of the tendons and ligaments and determine exactly where the injury has occurred. Surgical and regenerative treatments are important repair techniques for severed tendons and ligaments. The surgical procedure is performed with the animal standing or in lateral recumbency under general anesthesia, local anesthesia or sedation. Regular hoof trimming and proper shoeing should be applied; this will help the animal to keep good conformation and can help to relieve abnormal stress on the animal's tendons and ligaments.
\end{abstract}

Keywords: Ligaments; Regenerative Treatments; Surgical Treatments; Tendons

Abbreviations: AAEP: American Association of Equine Practitioners; AT-MSCs: Adipose Tissue Derived Mesenchymal Stem Cells; BM-MSCs: Bone Marrow Derived Mesenchymal Stem Cells; ECM: Extra Cellular Matrix; MPCs: Mesenchymal Progenitor Cells; MSCs: Mesenchymal Stem Cells

\section{Introduction}

Tendons and ligaments are similar dense fibrous connective tissues that connect skeletal muscle to the skeletal elements (bone) and bone to bone, respectively. Tendons serve primarily to transfer the pull of muscles to bone while ligaments connect one bone to another. Both are characterized by their great tensile strength and are dominated by collagen. However, tendons and ligaments have dynamic characteristics that lie their appearance- they are capable of repair after injury and respond to exercise or immobilization by altering their tensile strength. This dynamic behavior suggests that the cells in tendons and ligaments are capable of detecting changes in mechanical load and coordinating their response to alter the composition of the extracellular matrix (ECM) Frank [1]. Tendons and ligaments are composed of fiber like connective tissue elements that are carefully aligned in longitudinal bundles that run in the direction of force or pull in the entire structure. These bundles of fibers are grouped together, beginning in small units, and then combined with others to form larger and larger parallel fiber bundle groups, much like the structure of a cable on a bridge. Both tissues are also characterized by the presence of fibroblasts/fibrocytes (ligament) or tenoblasts/tenocytes (tendon) and an abundant extracellular matrix (ECM), mainly composed of collagen I Gelse et al. [2], resulting in a dense and hypo cellular structure. Tendon and ligament tissues have a pivotal mechanical role in joint stability, due to their poor natural healing capacity. Injuries in these tissues severely affect the joint functionality, which can ultimately result in the progression of degenerative diseases Fleming et al. [3].

The main function of ligaments and tendons is to transmit tensile loads. In experimental studies, tensile testing of these tissues results in a nonlinear load elongation curve that enables them to maintain smooth movement of joints under normal circumstances and to restrain excessive joint displacements under high loads. From the same test, the mechanical properties of the ligament or tendon substance, represented by the stress-strain curve, can also be obtained Woo et al. [4]. Tendon and ligaments are low vascularized tissues and have low healing capacity and capability; therefore management of tendon and ligament injuries is technically de- 
manding Moshiri \& Oryan [5]. Tendon and ligament injuries vary in nature and could mainly be divided into acute and chronic injuries. In some of the acute tendon or ligament injuries, a simple transaction is occurred as a result of sharp traumas or during routine orthopedic surgeries (e.g. bone fixation using plating techniques; tissue dissection, etc.).

In addition, these types of injuries have been reported to occur as a result of vehicular trauma, or due to falling from hill. Basically, it is a rule to reappose the transected tendon edges and sutures them Park \& Sung [6]. The most commonly damaged tendons and ligaments are those that run down the back of the limb from the stifle/elbow to the hoof (the superficial digital flexor tendon, the deep digital flexor tendon, the accessory ligaments and the suspensory ligament). The flexor tendons help flex the fetlock and the digit, while the suspensory ligament provides support for the fetlock, preventing extreme over-extension Mascarenhas \& Macdonald [7]. Forelimb suspensory ligament injuries are common in horses that have to brake quickly from high speeds and perform quick forelimb turns, such as barrel racers and polo ponies. Racehorses, show jumpers, and eventers most commonly injure the superficial digital flexor tendon in the front limbs. Dressage horses are more prone to suspensory ligament strains, especially when working in deep sand areas Pankaj and Nicola [8].

Regardless of the acute or chronic nature of the tendon and ligament injuries, surgical reconstruction of the large and massive tissue defects is a more complicated situation. There are some conventional available options in managing such large tissue losses including auto- allo- and xenografts. Autografts are still gold standard while the allografts and xenografts have been introduced as alternative options having lower value than the autografts. All these available options have their own significant limitations. Tissue engineering is a newer option and is divided into four categories of scaffold, healing promotive factors, stem cells and gene therapy Park and Sung [6]. Therefore, the objective of this review is to highlight the tendon and ligament repair techniques.

\section{Causes, Signs and Diagnosis of Tendon and Ligament Injuries}

Most tendon strains are associated with excessive loading and over stretching of the tendon due to excessive physical stress (such as occurs when a horse lands a jump). As the tendon is stretched beyond its normal capacity, the collagen fibers within it begin to tear and some may rupture. Though the tendon fibers can tear all at once, for example when a horse is galloping and steps in a hole, these injuries are most often caused by repetitive stress during training and events, when minor disruption or tearing of the tendon fibers take place day after day. Tendon injuries can also be caused by external trauma. Hard blunt trauma can cause significant injury; examples are a leg being caught under a fence plank or a horse brushing one leg with another Frank [1].

Tendon injury (or tendonitis) is characterized by inflammation with hemorrhage, edema and fibrin accumulation within and around the tendon, causing local swelling. The net result of the inflammation created by the tendon fiber tearing is a series of events that further damage and weaken the surrounding fibers Riley [9]. As the maximum strain limit is exceeded, the collagen fibers and blood vessels within the core of the tendon begin to rupture. The end result of tendons and ligaments injuries are inflammation, pain, Lameness, Heat, Swelling (typical 'bowed' appearance) and Pain on touch Benjamin \& Ralphs [10]. Damaged tendon and ligament can be diagnose by observing clinical signs like pain, swelling, lameness and Ultrasound is an excellent diagnostic tool. Ultrasound is an excellent diagnostic tool that allows veterinarian to view the fiber pattern of the tendons and ligaments and determine exactly where the injury has occurred. They can also determine whether the injury is mild, moderate or severe-this is important information needed to formulate a proper rehabilitation plan. Normal tendons and ligaments show a homogenous echogenic (evenly white) appearance on ultrasound when viewed on cross-section. Normal tendons and ligaments demonstrate a long linear fiber pattern. Injuries show up as increased cross-sectional areas (size) with decreased echogenicity (black or gray appearance and a disrupted fiber pattern Frank [1].

\section{Surgical Repairs of Severed Tendon and Ligament Desmotomy}

\section{Anesthesia and Surgical Preparations}

The surgical procedure is performed with the animal standing or in lateral recumbency under general anesthesia, local anesthesia or sedation. The area of ligament is clipped and surgically prepared. Two milliliters is of local anesthetic are injected subcutaneously over the ligament. A 20- gauge needle is then inserted through the bleb, and the subcutaneous area around the ligament is infiltrated with local anesthetic Singh et al. [11].

\section{Surgical Technique}

Skin incision is made and then a $1 \mathrm{~cm}$ incision is made over the ligament with a blunt-ended bistoury (tenotomy) knife. Curved Kelly forceps are then forced through the heavy fascia and are passed beneath the ligament. This creates a channel for the insertion of a bistoury knife beneath the ligament. The bistoury knife is inserted so the side of the knife lies flat beneath the ligament. When the knife is positioned, the cutting edge is then turned outward. With the left index finger palpating the end of the knife through the skin to ascertain its correct position, the surgeon cuts the ligament with a sawing movement. One or two sutures of non absorbable materials are placed in the skin incision Ditsios et al. [12].

\section{Postoperative Management}

Tetanus prophylaxis is administered. Bandaging is generally required for 2 to 3 weeks and sutures are removed 2 to 3 weeks post operatively. Box -stall rest is indicated until the surgical sites are healed. When the sites are healed, hand walking is commenced for about 2 weeks. After this period of hand walking, normal training is resumed Dahlgren [13].

\section{Tenotomy}

\section{Anesthesia and Surgical Preparation}

The surgery can be performed under local anesthesia with the animal standing or under general anesthesia. The local anesthetic 
should be injected in to the muscle where attached to the tendon. The area over the surgical site is clipped and shaved Capdevila \& Choquet [14].

\section{Surgical Technique}

An incision is made directly over the tendon; the tendon is exposed and isolated by dissecting bluntly beneath the tendon and elevating it using either curved Kelly forceps or oschner forceps. Pulling on the tendon at this stage reveals movement of the corresponding muscle; this will assist the surgeon in locating the incision over the muscle. The incision should continue through the overlying fascia until the fleshy portion of the muscle visible. The fascia overlying the muscle is thick and the fibers are directed diagonally. Once the muscle is freed, the surgeon goes to the first incision over the distal aspect of the tendon and severs the tendon. Prior to severing the tendon, one should make sure that the tendon in the distal incision corresponds to the muscle in the proximal incision Dowling et al. [15]. Then a pair of oschner forceps is placed under the musculo-tendinous junction; by exerting traction on it, the entire tendon is stripped from its sheath. The muscle is closed with simple horizontal mattress using a synthetic absorbable material. The fascia is closed with simple interrupted sutures of an absorbable suture material, followed by closure of the subcutaneous tissue with a similar material in a simple interrupted or continues pattern. The skin is closed with a synthetic, monofilament, nonbsorbable suture material in a simple interrupted pattern Ditsios et al. [12].

\section{Postoperative Management}

The wounds are covered with non adherent dressings and the entire limb is bandaged. Bandaging is generally required for 2 to 3 weeks and sutures are removed 2 to 3 weeks post operatively. Box -stall rest is indicated until the surgical sites are healed. When the sites are healed, hand walking is commenced for about 2 weeks. After this period of hand walking, normal training is resumed Dahlgren [13].

\section{Suture Patterns}

\section{Locking-Loop Tendon Suture (Modified Kessler Pattern)}

The needle is inserted into the severed end of the tendon and emerges from the surface of the tendon. The needle is then passed transversely through the tendon just superficial to the longitudinal part of the suture. This results in a loop of suture locking around a small bundle of tendon fibers. When more tension is applied to the repair site, the grip of the suture loop on these fiber bundles becomes tighter. The needle is then reinserted in a longitudinal direction and passes under the transverse portion of the suture material; this process is repeated on the other piece of tendon. After placement of the suture, all the loops should be tightened in turn and the suture tied snugly, so slight bunching occurs at the junction simon \& Wayne [16]. Monofilament nylon or polypropylene is the recommended suture material for this suture. Rough surface sutures do not have sufficient glide or elasticity to permit longitudinal strain to be transmitted in to locking tension. Wire is not flexible enough for this suture pattern and is not recommended. The largest diameter monofilament non absorbable suture should be used. At this time, the largest commercially available suture material of this type is no 2 nylon simon \& Wayne [16].

\section{Tsuge Suture Pattern}

Tsuge suture is designed for and used in three steps using a single-stranded suture monofilament polypropylene (Prolene). In the first step, the needle was laterally inserted into the proximal tendon end of the volar surface, with in $1 \mathrm{~cm}$ from the intended repair site. The strand was then run longitudinally across the repair conjuncture and taken out $1 \mathrm{~cm}$ away from the repair site at the distal tendon end. With the needle passed transversely in the distal part, the strand was taken across the loop and the suture reinserted into the distal tendon end, crossing the repair site at the dorsal surface and existing from the proximal end dorsally, so that it could be reintroduced transversely to make a loop. The second step involved the same procedure as the first step but exerted in the opposite side. To complete the third step, the peripheral suture was placed as running suture with monofilament polypropylene Chen et al. [17].

\section{Regenerative Treatments of Severed Tendon and Ligament Regenerative Medicine}

The aim of regenerative medicine is to restore the original architecture and biomechanical function to damaged tissues. Knowing the molecular and mechanic processes involved in the development of a tendon or ligament lesion and the pathologic conditions of the lesion itself (acute/chronic) allows to a better choice in the most appropriate therapy to use. There are 3 fundamental instruments to obtain a good regeneration of the damaged tendon : first, an implant compatible with the attachment and the migration of cells able to lead the reconstruction of the damaged area in a three-dimensional fashion; second, a source of cellular precursors able to recruit endogenous stem cells possibly differentiating in tenocites; third, a physiologic combination of growth factors and cytokines to make the tendon matrix reorganize on the given implant Fortier \& Smith [18].

\section{Stem Cells}

They are undifferentiated cells able to undergo differentiation in a specific cellular line and renovate. Mesenchymal stem cells (MSCs) are adult stem cells that can be isolated from bone marrow, muscle, tendon, adipose tissue, and can differentiate in cells such as osteoblasts, myocytes, chondrocytes, adipocytes, and tenocytes, depending on the tissue they have been implanted in. Adult stem cells can thus conduct a regenerative response; furthermore they contribute to healing producing bioactive proteins (growth factors and cytokines) sending local signals through the recruitment of endogenous stem cells; also they induce anabolic effects in the recruited cells and in those already present in the tissue. Stem cell therapy can help many injuries but is most commonly used to help heal tendon, ligament, bone and joint injuries. The therapy involves gathering stem cells from a source (bone marrow or fat), processing it to concentrate the stem cells and injecting it into an injury. Now a day's stem cells are applied in the form of a suspension inside the lesions of tendons, ligaments and joints Dahlgren [13]. 


\section{Bone Marrow Derived Mesenchymal Stem Cells (BM- MSCs)}

The use of these cells has been experimented in laboratories on animals previously submitted to surgery to create a lesion. These studies have reported positive effects on the organization of the tissue, on the composition and the mechanic properties of treated tendons and ligaments. MSCs are used in tissue engineering, in genic therapy or they are directly injected into the lesion. Among these possibilities only the direct injection is applicable in clinical practice. The injection of MSCs derived from bone marrow in tenodesmic lesions offers two great advantages:

a) MSCs can differentiate in mature fibroblasts able to produce new matrix;

b) Bone marrow is rich in blood thus it is a powerful source of growth factors that favor the healing of the tissue. The treatment can either consist in the injection of the entire marrow aspirate, of a concentrate of stem cells obtained with centrifugation or of a population of stem cells cultured in vitro Fortier and Smith [18].

\section{Adipose Tissue Derived Mesenchymal Stem Cells (AT- Mscs)}

The use of adipose tissue as source of adult stem cells offers numerous advantages compared to bone marrow:

a) The collection technique is easier and less invasive;

b) Stem cells are more concentrated and the amplification in vitro is not necessary;

c) These stem cells show a higher proliferative rate in vitro. The population of stem cells isolated from adipose tissue is heterogeneous and a physiologic combination of fibroblasts, endothelial precursors, pericytes, macrophages, B and T lymphocytes, pre-adipocytes and smooth muscle cells Dahlgren [13].

\section{Grafts}

In severe damage, biological grafts may be needed to replace damaged tendons. Nevertheless, in most cases, grafts do not provide adequate mechanical strength during the remodeling process. Auto grafts are used to repair the affected tendon, preventing instability and reducing the rate of rupture. Auto grafts may also lead to high morbidity and functional disability at the donor site and limited availability. Tissue laxity, mechanical mismatch and poor tissue integration (with a possible necrosis after implantation) are also disadvantages to be considered. Allografts are also therapies for tendon and ligament repair but are not ideal, due to the associated risk of disease transmission and tissue rejection Curwin [19]. Tendon grafts, whether autogenous or allogenic, undergo a more or less similar process of integration characterized by graft necrosis, revascularization, and cell repopulation and remodeling. The process of allograft integration is significantly affected by the method of tissue processing. The fresh allograft tissue is not suitable for implantation because it is highly immunogenic. Allograft tissues significantly reduce the immunogenicity of the tissue by killing fibroblasts. These processes remove the loci for the major histocompatibility antigens, allowing allografts to be used even in immunologically incompatible hosts without provoking a significant immune response Robertson et al. [20].

In complex ligament injuries, tendon allograft provides a distinct advantage over autograft because of the limited availability of intact host tissue, allograft are pretty simple. Using an allograft tendon will allow the patient to avoid donor site morbidity, reduces surgical time, smaller incisions, availability of larger grafts and no weakening of the extensor or flexor apparatuses. Allograft reconstruction also provides a safer alternative to those patients who are at higher risk of complications under anesthesia. Considering that the surgeon would not have to take time to harvest and prepare the autograft, he would be able to complete the reconstruction faster thus avoid having the patient under for longer periods of time. The specific concerns related to allograft are tissue availability, sterilization, graft cost, delayed graft incorporation, disease transmission, and long term graft strength Miller \& Gladstone [21]. Autograft plays an important role in reconstruction of the extensively damaged tendon and ligament in a number of anatomical sites as it provides a long and wide aponeurosis.

The advantage of autograft ligament reconstruction is no risk of disease transmission. The animal body tends to accept its own tendons better than tendons from a donor. The fact that there is no risk of disease transmission is a very big positive for autograft Alam et al. [22]. The use of autogenous tissue for patellar ligament replacement has many advantages over the use of allografts. Autogenous tissue will not be rejected by the body, negating the need for immune suppression. There is no requirement for additional procedures to harvest grafts from donors and no need for storage facilities. Fascialata has been successfully employed as a free graft for Achilles mechanism reconstructions Smith [18]. A potential disadvantage of fascia lata is its reduced static strength compared to intact patella tendon. It is therefore necessary to protect the graft during the healing process using encircling sutures and some form of external support, and to restrict the patient's exercise in the postoperative period Gemmill \& Carmichael [23,24].

\section{Conclusion and Recommendations}

The most commonly damaged tendons and ligaments are those that run down the back of the limbs from the stifle/elbow to the hoof (the superficial digital flexor tendon, the deep digital flexor tendon, the accessory ligaments and the suspensory ligament) and these severed tendons and ligaments can be repaired by surgical or regenerative treatments.

Based on the above conclusion, the following recommendations are forwarded:

a) Regular hoof trimming and proper shoeing should be applied; this will help the animal to keep good conformation and can help to relieve abnormal stress on the animal's tendons and ligaments. 
b) Proper area should be constructed; uneven, slippery ground or sand may disproportionately load one side of a tendons and ligaments, causing excessive physical stress to them.

c) Long training sessions or hard workouts should be avoided; muscle exhaustion/fatigue from long training sessions or hard workouts results in poor muscle response and loss of tendons and ligaments stabilization.

\section{References}

1. Frank B (2004) Ligament structure, physiology and function. Journal of Musculoskeletal and Neuronal Interactions 4(2): 199-201.

2. Gelse, K, Poschl E, Aigner T (2003) Collagens structure, function, and biosynthesis. Advanced Drug Delivery Reviews 55(12): 1531-1546.

3. Fleming C, Hulstyn M, Oksendahl H (2005) Ligament injury, reconstruction and osteoarthritis. Current opinion in orthopedics 16(5): 354-362.

4. Woo Y, Gomez A, Seguchi Y, Endo M, Akeson H (1983) Measurement of mechanical properties of ligament substance from a bone-ligamentbone preparation. Journal of Orthopaedic Research 1(1): 22-29.

5. Moshiri A, Oryan A (2012) Role of tissue engineering in tendon reconstructive surgery and regenerative medicine: current concepts, approaches and concerns. Hard Tissue 1: 11.

6. Park S, Sung K (2012) Surgical reconstruction of chronic achilles tendon ruptures using various methods. Orthopedics 35(2): 213-218.

7. Mascarenhas R, Macdonald B (2008) Anterior cruciate ligament reconstruction: a look at prosthetics - past, present and possible future. McGill Journal of Medicine 11(1): 29-37.

8. Pankaj S, Nicola M (2005) Tendon Injury and Tendinopathy: Healing and Repair. The Journal of Bone and Joint Surgery 87(1): 187-202.

9. Riley G (2004) The pathogenesis of tendinopathy. A molecular perspective. Rheumatology (Oxf) 43(2): 131-142.

10. Benjamin M, Ralphs R (2000) The cell and developmental biology of tendons and ligaments. International Review of Cytology 196: 85-130.

11. Singh K, Gangwar A, Devi S, Singh N (2015) Studies on incidence and evaluation of the closed medial patellar desmotomy in lateral recumbency in bovines. Veterinary World 8(2): 221-224.

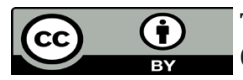

This work is licensed under Creative Commons Attribution 4.0 License

Submission Link: https://biomedres.us/submit-manuscript.php
12. Ditsios T, Burns E, Boyer I, Gelbermann H, Silva J (2002) The rigidity of repaired flexor tendons increases following ex vivo cyclic loading. Journal of Biomechanics 35(6): 853-856.

13. Dahlgren A (2009) Management of Tendon Injuries. Current Therapy in Equine Medicine ( $6^{\text {th }}$ edn), Saunders 30: 518-523.

14. Capdevila X, Choquet O (2008) Regional Anesthesia and Patient Outcomes. Techniques in Regional Anesthesia and Pain Management 12(4): 161-210.

15. Dowling A, Dart J, Hodgson R, Smith W (2000) Superficial digital flexor tendonitis in the horse. Equine Veterinary Journal 32(5): 369-378.

16. Simon A, Wayne $T$ (1989) Techniques in large animal surgery. $\left(2^{\text {nd }}\right.$ Edition) 12: 107-108.

17. Chen J, Wang K, Katirai F, Chen Z (2014) A new modified Tsuge suture for flexor tendon repairs: the biomechanical analysis and clinical application. Journal of Orthopedic Surgery and Research 9: 136.

18. Fortier A, Smith W (2008) Regenerative Medicine for Tendinous and Ligamentous Injuries of Sport Horses. Veterinary Clinics Equine 24(1): 191-201.

19. Curwin L (2005) Rehabilitation after tendon injuries. In Tendon Injuries: Basic Science and Clinical Medicine. Springer-Verlag: London, UK 22: 242-266.

20. Robertson A, Nutton R, Keating J (2006) Current trends in the use of tendon allografts in orthropaedic surgery. Journal of Bone and Joint Surgery 88(8): 988-992.

21. Miller L, Gladstone N (2002) Graft selection in anterior cruciate ligament reconstruction. The Orthopedic clinics of North America 33(4): 675-683.

22. Alam M, Gordon W, Heo S, Lee K, Kim N (2013) Augmentation of ruptured tendon using fresh frozen Achilles tendon allograft in two dogs: a case report 58: 50-55.

23. Gemmill T, Carmichael S (2003) Complete patellar ligament replacement using a fascia lata autograft in a dog. Journal of Small Animal Practice 44: 456-459.

24. Smith R (2008) Tendon and Ligament Injury, Proceedings the American Association of Equine Practitioners 54: 475-501.

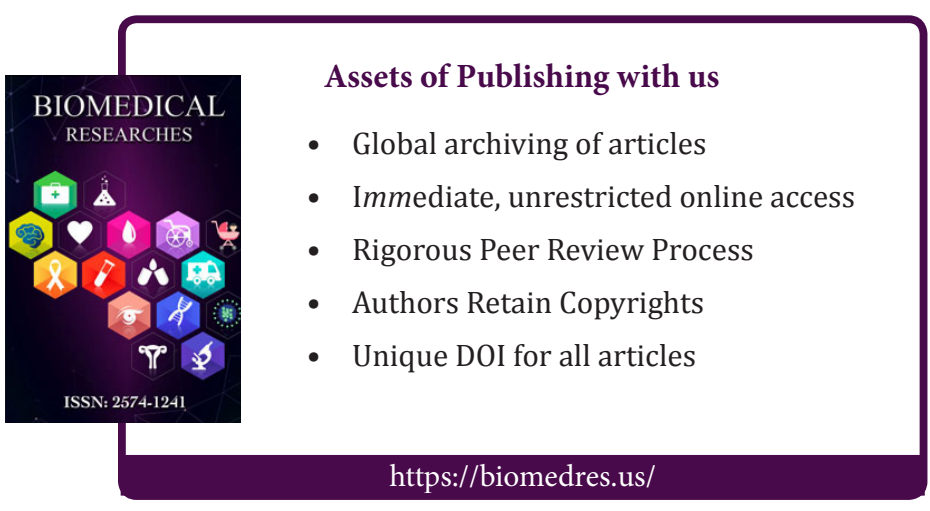

